\title{
Environmental Factors Predict the Severity of Delirium Symptoms in Long-Term Care Residents with and without Delirium
}

Jane McCusker, MD, DrPH, ${ }^{\mathrm{a}, \mathrm{b}}$ Martin G. Cole, MD, ${ }^{\mathrm{a}, \mathrm{d}, \mathrm{d}}$ Philippe Voyer, RN, PhD, ${ }^{\mathrm{e}}$ Minh Vu, MD, ${ }^{\text {f,g }}$ Antonio Ciampi, PhD, ${ }^{\mathrm{a}, \mathrm{b}}$ Johanne Monette, MD, MSc, ${ }^{\mathrm{h}}$ Nathalie Champoux, MD, MSc, ${ }^{\mathrm{i}}$ Eric Belzile, MSc, ${ }^{a}$ and Alina Dyachenko, $\mathrm{MSc}^{\mathrm{a}}$

${ }^{a}$ St. Mary’s Research Centre, St. Mary’s Hospital, Montreal, Canada; Departments of ${ }^{b}$ Epidemiology, Biostatistics, and Occupational Health and ${ }^{\mathrm{d}}$ Psychiatry, McGill University, Montreal, Canada

${ }^{\mathrm{c}}$ Department of Psychiatry, St. Mary’s Hospital Center, Montreal, Canada

${ }^{\mathrm{e}}$ Faculty of Nursing Sciences, Laval University, Sainte-Foy, Canada

${ }^{\mathrm{f}}$ Division of Geriatric Medicine, Centre Hospitalier de l’Université de Montréal, Montreal, Canada

${ }^{\mathrm{g}}$ Department of Medicine, Université de Montréal, Montreal, Canada

${ }^{\mathrm{h}}$ Division of Geriatric Medicine, Jewish General Hospital and Maimonides Geriatric Center

IInstitut Universitaire de Gériatrie de Montréal, Department of Family Medicine, Université de Montréal, Montreal, Canada

Corresponding author: Dr. J. McCusker, St. Mary’s Research Centre, Hayes Pavilion, Suite 3734, 3830 Avenue Lacombe, Montreal, Quebec, H3T 1M5, Canada. E-mail: jane.mccusker@mcgill.ca

This work was funded by: the Canadian Institutes for Health Research, Institute of Aging (IAO 69519); the Alzheimer Society of Canada with the Canadian Nurses Foundation and the Nursing Care Partnership of the Canadian Health Services Research Foundation (ASC 0791); the Canadian Institutes for Health Research Institutes of Aging and of Gender and Health (CRG 82953); and the Canadian Institutes for Health Research, Institute of Aging with the Alzheimer Society of Canada and the Canadian Nurses Foundation (CPC 102348).

Objectives: To identify potentially modifiable environmental factors (including number of medications) associated with changes over time in the severity of delirium symptoms and to explore the interactions between these factors and resident baseline vulnerability.

Design: Prospective, observational cohort study.

Setting: Seven long-term care (LTC) facilities.

Participants: Two hundred seventy-two LTC residents aged 65 and older with and without delirium.

Measurements: Weekly assessments (for up to 6 months) of the severity of delirium symptoms using the Delirium Index (DI), environmental risk factors, and number of medications. Baseline vulnerability measures included a diagnosis of dementia and a delirium risk score. Associations between environmental factors, medications, and weekly changes in DI were analyzed using a general linear model with correlated errors. 
Results: Six potentially modifiable environmental factors predicted weekly changes in DI (absence of reading glasses, aids to orientation, family member, and glass of water and presence of bed rails and other restraints) as did the prescription of two or more new medications. Residents with dementia appeared to be more sensitive to the effects of these factors.

Conclusion: Six environmental factors and prescription of two or more new medications predicted changes in the severity of delirium symptoms. These risk factors are potentially modifiable through improved LTC clinical practices.

Key words: aged, long-term care, delirium, risk factors

Delirium ranges in prevalence from 3.4\% to 70.3\% in long-term care (LTC) and postacute care facilities. ${ }^{1-8}$ In postacute settings, delirium is a risk factor for mortality, ${ }^{8,9}$ and in LTC, it is a risk factor for adverse outcomes, ${ }^{1}$ so efforts are warranted to prevent delirium in LTC settings.

In acute-care settings, precipitating factors (e.g., physical restraints, >3 medications added) interact with predisposing factors (e.g., presence of dementia) such that precipitating factors are more likely to lead to delirium in individuals with greater baseline vulnerability. ${ }^{10}$ The LTC population differs in important respects from the acute care population (e.g., greater prevalence of dementia and functional impairment, less-acute medical illness, long-term residence, different type and frequency of risk factors, greater use of psychoactive medications). ${ }^{7}$ Consequently, potentially modifiable risk factors for delirium may differ from those in acute care. ${ }^{7}$ The role of these risk factors needs to be investigated in residents with different levels of vulnerability.

The current study investigated environmental factors and medications associated with changes over time in severity of delirium symptoms. This approach allowed factors that lead to worsening and improvement in delirium symptoms to be investigated and allowed for a more-comprehensive evaluation of risk factors than one based only on predicting incident delirium. A similar methodology in acute care settings identified environmental factors and medications that were related to changes in the severity of delirium symptoms. 11,12

This study was conducted in LTC residents aged 65 and older with cognitive impairment ranging from none to severe recruited in seven facilities and followed weekly for up to 6 months. It has been reported that delirium symptoms occur frequently in this population for extended periods during episodes of delirium, before and after episodes of delirium, ${ }^{13}$ and in residents who never had an episode of delirium during the observation period. ${ }^{14}$ Therefore, delirium symptoms were studied in all residents whether or not they met criteria for delirium. The objectives of this study were to identify potentially modifiable environmental factors that predict changes over time in the severity of delirium symptoms 
and to explore the interactions between these potentially modifiable factors and measures of resident baseline vulnerability, including dementia and a delirium risk screening score ${ }^{15}$.

\section{METHODS}

The data source for this study, well described previously, ${ }^{1,7}$ was a prospective, observational, cohort study of LTC residents at seven study sites in Montreal and Quebec City. Residents aged 65 and older admitted for LTC were recruited. Stratified sampling with two strata were used: no to moderate cognitive impairment (Mini-Mental State Examination (MMSE) score $\geq 10$ ) and severe cognitive impairment (MMSE score $<10$ ). Newly admitted and longer-term residents were recruited consecutively from resident lists. Residents were excluded only if they were unable to communicate in English or French or the primary nurse or research assistant (RA) did not have time to complete assessments because of a high workload. Competence to consent to the study was based on the clinical impression of the primary nurse. A trained RA invited competent residents to participate in the study. For incompetent residents, a letter describing the study was sent to the legal guardian if available or (because many LTC residents are not legally declared incompetent) to the responsible family member. The legal guardian or family member informed the nurse if they were willing to meet the RA. The research ethics boards of McGill University and sites with a review committee approved the study protocol.

The RA assessed the residents weekly for up to 24 weeks or until death or transfer to another facility. The primary nurse (who was blind to the RA assessments) was interviewed at baseline.

\section{Outcome measure}

The outcome, severity of delirium symptoms, was measured weekly using the Delirium Index (DI). Adapted from the Confusion Assessment Method (CAM) ${ }^{16}$, the DI is a measure of the severity of delirium that a trained RA (nonpsychiatrist) can score on the basis of observation, without additional information from family members, nursing staff, or the individual's medical chart. ${ }^{17}$ The DI has an excellent level of interrater reliability and good construct validity and is responsive to change over time in individuals with delirium. ${ }^{18}$ It includes seven of the 10 symptom domains of the CAM (disorders of attention, thought, consciousness, orientation, memory, perception, and psychomotor activity), each scored on a scale from 0 (absent) to 3 (severe) using operational criteria for each score. Thus, the total DI score may vary from 0 to 21, a higher score indicating greater severity. The other three domains of the CAM (acute onset, fluctuation, sleep-wake disturbance) are not included in the DI because they do not assess severity (acute onset, fluctuation) or cannot be assessed easily in a single observation period (sleep-wake disturbance).

To strengthen support for use of the DI as a measure of severity of delirium in the LTC context, cases of probable delirium (CAM criteria) are also reported on, and DI scores were computed for residents with delirium and CAM core symptoms of delirium not 
meeting criteria for delirium (the presence of $\geq 1$ of inattention, fluctuation, disorganized thinking, altered level of consciousness.)

The DI score was prorated when only one or two symptoms were missing (2.4\% with 1 missing, $0.5 \%$ with 2 missing). The study psychiatrist (MC), who conducted periodic interrater reliability sessions, supervised the RAs. Three raters simultaneously performed 28 assessments; the interclass correlation coefficient (ICC) was calculated as a measure of interrater reliability, and an ICC of 0.90 (95\% confidence interval $(\mathrm{CI})=0.80-0.95)$ was obtained.

\section{Baseline measures}

At baseline, demographic variables and several measures of baseline vulnerability were measured: the Delirium Risk Screening Score (developed from the same cohort), ${ }^{15}$ severity of cognitive impairment, diagnosis of dementia, and overall comorbidity. Severity of cognitive impairment was assessed using the MMSE and the Hierarchic Dementia Scale (HDS). The RA rated the MMSE, ${ }^{19,20}$ validated for use in LTC facilities ${ }^{21}$; MMSE scores range from 0 to 30, a lower score indicating greater cognitive impairment. The HDS ${ }^{22}$ is a scale that can be used in individuals with mild or severe cognitive impairment ${ }^{23}$ and has been validated in LTC residents. ${ }^{24}$ Total HDS scores range from 0 to 200 and were grouped into four severity categories (severe (0-39) moderate (40-99), mild (100-159), minimal to none $(\geq 160)$ ). Medical charts were used to extract information on sociodemographic variables and medical problems. Duration of residence at the facility was grouped into two categories ( $<12 \mathrm{vs} \geq 12$ months). Medical problems and diagnoses were extracted from medical charts for the period between admission and the baseline interview. These were used to measure a diagnosis of dementia and to compute the Charlson Comorbidity Index (CCI), ${ }^{25,26}$ validated as a predictive index for survival in two nursing home cohorts. ${ }^{27,28}$

\section{Environmental factors and medications}

Ten environmental factors were defined on the basis of observations that the RAs made at weekly assessments (absence of personal belongings (e.g., family pictures), understimulation (inadequate lighting or sound level), absence of aids to orientation (e.g., calendar, clock, or watch), uncorrected visual impairment (visually impaired residents not using reading glasses), uncorrected hearing impairment (hearing-impaired residents not using hearing aids), bed rails, other physical restraints (lap belt, chair -table, or handrestraints), no glass of water within reach of resident, absence of a family member, and absence of a staff member visible from door of resident's room). Room changes and changes in location of each assessment were also examined. Room changes were rare in this population and were not analyzed further. Changes in location of each assessment (in the resident's room or elsewhere) had no effect on the outcome and were not considered further.

Interrater reliability of the assessments of the 10 environment risk factors was assessed in eight sessions over the course of the study. In each session, one to four residents were observed for a total of 22 assessments. In each assessment, three RAs observed the 
environment for presence or absence of a risk factor; the three observers were considered in agreement if their reports coincided. There was good overall agreement (75-100\%) for presence of a family member, personal belongings, bed rails, and other restraints. Overall agreement levels were moderate (64\%) for understimulation, visibility of a staff member, and presence of a glass of water and lower for uncorrected visual and hearing impairment (50-53\%) and aids to orientation (50\%). Further inspection of these data revealed much lower agreement for visual and hearing impairment during the two sessions that comprised six assessments with residents with very severe cognitive impairment (MMSE score 0); after exclusion of these sessions, there was better agreement (75\% and 69\%, respectively). The ratings of aids to orientation improved substantially over the course of the study to $71 \%$ in the last seven assessments, with greater focus on standardization of measures.

Using the provincial drug number, a single coder coded prescribed medications (excluding topical medications and nutritional supplements), which were summed to give a total daily number of medications. Daily numbers of psychotropic and nonpsychotropic medications were also examined.

\section{Nonmodifiable time-dependent risk factors}

Data were extracted from resident charts for vital signs, laboratory test results, chest X-rays, new medical problems, nursing notes on physical and functional changes, emergency department visits, and hospital admissions (Appendix 1).

\section{Statistical methods}

Descriptive statistics were computed for each risk factor and outcome (Table 2). First, for each resident, the longitudinal binary and continuous variables were summarized over all available time points using proportions and means, respectively. Then, means, standard deviations, and medians with first and third quartiles were computed among all residents.

Change in the outcome was defined as difference in DI score between two consecutive weekly assessments $(\mathrm{t}-1, \mathrm{t})$. Four categories of weekly change in each environmental risk factor were defined: continued exposure (the risk factor was present at $\mathrm{t}-1$ and $\mathrm{t}$, decreased exposure (the risk factor was present at $\mathrm{t}-1$ but not at $\mathrm{t}$, increased exposure (the risk factor was not present at $t-1$ but present at $t$, and no exposure (the risk factor was absent at $\mathrm{t}-1$ and $\mathrm{t}$ ). The last was used as the reference category. Missing exposure at $\mathrm{t}-1$ or $\mathrm{t}$ was treated as an additional category when the research assistant was not able to assess the environmental checklist item. In the case of other restraints, a change in type of restraint (e.g., from lap table to hand restraints) from t-1 to t was considered to be an increase in exposure. In the case of medications, exposure was assessed during the week before each assessment (the weeks before $t-1$ and $t$, respectively), and the average daily number of medications was computed.

The relationship between outcome and risk factors was investigated using a general linear model ${ }^{29}$ (GLM) with correlated errors to account for the repeated measures on individual patients; selection of the error covariance structure was based on stability of convergence (only models that did not exhibit numerical instabilities in the estimation 
procedures were used) and on the Bayesian Information Criterion (BIC) and Akaike Information Criterion (AIC). ${ }^{29}$ It was concluded that a GLM with first-order autoregressive structure fitted the data sufficiently well. Regression coefficients (betas) with their 95\% CIs were computed for each risk factor exposure category in comparison with the reference category. Each risk factor was adjusted for time (by adding the time variable measured in weeks as a continuous predictor) and DI score at time $\mathrm{t}-1$ (Beta1). A full multivariable model was computed wherein baseline variables (age, sex, duration of residence, severity of dementia (HDS), diagnosis of dementia, CCI, Delirium Risk Screening Score, and severe cognitive impairment (MMSE $<10)$ ) and all the risk factors were also presented (Beta2). A similar approach was used to compute Beta1 for both predictors (environmental risk factor score and mean daily number of medication); otherwise, Beta2 was estimated separately for each predictor by adding baseline variables. To check for multicollinearity of predictors, the Variance Inflation Factor (VIF), ${ }^{30}$ an index considered to indicate potential multicollinearity if it takes values greater than 5 , was computed for each predictor. ${ }^{31}$

An environmental risk factor score was calculated as the number of selected environmental risk factors present for a resident at time t. Factors whose change was significantly associated with change in DI in one or more models were included (Table 3). When one or two risk factors were missing (6\% with one missing; $0.4 \%$ with 2 missing) the score was prorated (i.e., the score was computed as the mean of nonmissing items multiplied by the total number of items used in the score). Otherwise, a missing exposure category was defined when the score was missing at $\mathrm{t}-1$ or $\mathrm{t}$.

The nonmodifiable time-dependent risk factors were also adjusted for (Appendix 1). Although several of these factors were associated with changes in the DI, the addition of these risk factors to the multivariable models reported in Tables 3 and 4 had a minimal effect ( $<1 \%$ change in beta) on the effects of the environmental risk factors and medications, so the results of the adjustment are not reported here.

All the analyses were performed using SAS version 9.2 (in particular, PROC MIXED for GLM modeling; SAS Institute, Inc., Cary, NC).

\section{RESULTS}

Table 1 shows the characteristics of the study sample of 272 residents at baseline. Eight individuals were removed from the study sample because they did not have two consecutive weekly assessments. The majority were aged 80 and older, female, resident for at least 1 year in the facility, and diagnosed with dementia. Table 2 shows the average prevalence over time of the environmental factors of interest in the study sample. These ranged from $4 \%$ (absence of personal possessions) to 82\% (absence of a family member). The mean number of environmental factors per resident at one assessment was 3.2, and the mean number of daily medications was 6.7 .

During follow-up, the 272 residents met criteria for delirium at 538 assessments, for symptoms of delirium at 1,768 assessments, and for neither at 2,357 assessments. Mean DI 
scores were: 9.0 (95\% CI=8.5-9.5) for delirium, 7.6 (95\% CI=7.1-8.0) for symptoms of delirium, and 5.3 (95\% CI=4.8-5.7) for neither. One hundred ten residents (40.4\%) met criteria for probable delirium at least once during the study period; an additional 126 (46.4\%) had one or more core delirium symptoms at least once during the study period, before and after episodes of delirium or in the absence of an episode of delirium; and 36 (13.3\%) never had core delirium symptoms.

Table 3 shows the results of selected regression models for three types of exposure to the environmental risk factors of interest. Two models are shown for each type of exposure, a preliminary model that adjusts only for DI score at $\mathrm{t}-1$ and time (Beta1) and a multivariable model that adjusts also for all environmental factors and baseline variables (Beta 2). (Baseline variables were age, sex, duration of residence, diagnosis of dementia, Charlson score, and study design variable (MMSE score).) Increases in exposure to two risk factors (absence of reading glasses and a glass of water) were significantly associated with increases in DI score in both models. Absence of aids to orientation was significantly associated only with Beta1. Decreases in exposure to two risk factors (absence of reading glasses and absence of a family member) were significantly associated in both models with decreases in DI score. Continued exposure to four risk factors (absence of reading glasses, absence of aids to orientation, presence of other restraints, and absence of glass of water) was associated with an increase in DI score in both models. Continued absence of a family member was associated with change in DI but in a direction opposite from that expected. Continued presence of bed rails was significantly associated with increase in DI score only for Beta2. The proportions in the missing exposure category varied between $1 \%$ and $5 \%$, except for the absence of staff member (9\%), which was added 3 months after the beginning of the study. For each risk factor, the missing exposure category was not significantly associated with the outcome.

Based on these results, an environmental risk factor score was constructed that comprised the six environmental factors associated with any exposure type in the initial or multivariable model: absence of reading glasses, aids to orientation, a family member, and glass of water and presence of bed rails and other restraints. One point was assigned to each risk factor at each assessment time (possible range in scores 0-6). For Beta1 and Beta2 models, increase or decrease of two or more in environmental risk factor score was associated with a significant increase or decrease, respectively, in DI score (Table 4). An increase of 1 point was associated with a small increase in DI score only for Beta2. An increase of two or more medications was associated with an increase in DI score for both models, but there was no corresponding association with a decrease in number of medications. Whether increases in psychotropic or nonpsychotropic medications were responsible for the medication effect was explored using an increase of one or more medications in each category. These analyses indicated higher (but not significantly different) Beta2 estimates for psychotropic medications $(0.42,95 \% \mathrm{CI}=0.10-0.75)$ than for nonpsychotropic medications $(0.16,95 \% \mathrm{CI}=-0.01-0.33)$. 
Stratified analyses suggested that the environmental risk factor score and number of medications had a stronger effect on residents with a diagnosis of dementia (Table 4) but not a higher initial Delirium Risk Screening Score (data not shown). Because of the multicollinearity with diagnosis of dementia, the HDS and Delirium Risk Screening Scores were removed from all multivariate models presented in Tables 3 and 4.

\section{DISCUSSION}

This study identified six environmental factors that predicted change over time in severity of delirium symptoms in a cohort of LTC residents: absence of reading glasses, aids to orientation, a family member, and a glass of water and presence of bed rails and other restraints. Increases or decreases in two or more of these risk factors were associated with corresponding changes in DI score. An increase of two or more medications was also associated with an increase in delirium symptoms, but a decrease in the number of medications was not associated with a corresponding change. Residents with a diagnosis of dementia appeared to be more sensitive to the effects of these factors, in keeping with the vulnerability hypothesis proposed previously. ${ }^{10}$

The measure of the severity of delirium symptoms used — the DI—has been validated previously in acute care settings. Results of this study suggest that the DI is also a valid measure in LTC; the DI score differentiates three conditions-probable delirium, symptoms of delirium only, and neither and is responsive to changes in environmental risk factors and medications over time. ${ }^{17,18}$

Although environmental risk factors for delirium in LTC have previously been investigated only in cross-sectional studies, ${ }^{32,33}$ longitudinal studies in acute care settings have also found some of the same risk factors (absence of aids to orientation, absence of reading glasses, use of restraints, ${ }^{11}$ and increase in number of medications). ${ }^{10,12}$ The absence of family members had inconsistent effects: decreased exposure and continued exposure were associated with a decrease in the DI score. In a study in acute care, presence of a family member was associated with worsening of delirium severity. ${ }^{11}$ Further investigation is warranted of the effects of family members on the mental state of their relatives in LTC. Dehydration is a putative risk factor for delirium ${ }^{34}$; the findings of the current study suggest that placing a glass of water within easy reach may abate dehydration and reduce delirium risk in the LTC population. Three of the risk factors of interest were not associated with changes in DI: absence of hearing aids in those with hearing impairment, ${ }^{35}$ absence of personal possessions, and absence of sensory stimulation.

Changes in delirium symptoms were found only when there was an increase of two or more medications, similar to a finding in acute care. ${ }^{10}$ Psychotropic medications appeared to be more strongly associated with change in delirium symptoms than nonpsychotropic medications. These associations should be interpreted with caution because of the potential for confounding by indication or reverse causality. In-depth exploration of the complex 
relationships between types of medications, timing of exposure, and delirium symptoms was beyond the scope of this study and will be further investigated.

In general, the magnitude of the effects found in this study is modest and may not be clinically significant. For example, increases of two or more environmental risk factors or medications were associated (after adjustment for other factors) with an average increase of between 0.4 and 0.5 points on the DI score that ranges from 0 to 21 . Previous research in an acute care setting using this scale found that similar DI changes (0.4-0.5 points) were associated with an increase of one medication or absence of aids to orientation; stronger effects were found for uncorrected visual impairment (0.8 points) and physical restraints (1.2 points). ${ }^{11,12}$

There are at least six potential limitations of this study. First, weekly assessments may have missed interim changes in DI scores or environmental factors. Second, some of the risk factors had only moderate interrater reliability, but because the same RA usually assessed the same residents over time, the effect of this was probably small. Third, the associations between changes in the number of medications and delirium symptoms may have been due to changes in delirium symptoms associated with conditions (e.g., infections) for which medications (e.g., antibiotics) were prescribed (confounding by indication), although adjustment for medical status over time did not change these results. Fourth, the same RAs assessed environmental factors and delirium symptoms, although the RAs were not aware of the study hypotheses. Fifth, the analyses of certain risk factors with skewed distributions (absence of personal possessions, uncorrected hearing impairment) may have lacked statistical power. Sixth, because residents with and without delirium were included,

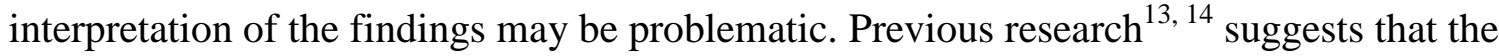
occurrence of delirium symptoms (not meeting criteria for delirium) reflects a delirium-like disorder - a prodrome or postdrome to an episode of delirium or subsyndromal delirium, both probably related to underlying medical conditions not severe enough to cause full delirium; changes in these symptoms related to environmental factors could then be understood in the same way as changes in symptoms of full delirium related to environmental factors in acute care settings. ${ }^{11,12}$ It is possible that delirium symptoms and changes in these symptoms in residents without delirium are part, albeit a poorly understood part, of the phenomenology of dementia in LTC residents. The findings were similar in residents with and without dementia, albeit more pronounced in residents with dementia. Further research is warranted on whether the occurrence of delirium symptoms (not meeting criteria for delirium) or changes in these symptoms should be attributed to delirium, a delirium-like disorder, or dementia.

These results support the implementation of good clinical practices in LTC settings that target specific environmental factors. ${ }^{36}$ Some factors are more easily modifiable than others. Provision of aids to orientation and water to all residents and ensuring that residents with reading glasses are using them are easier to implement. Visually impaired residents who do not have reading glasses may require optometry services. The use of bed rails and 
other restraints should be reviewed regularly for all residents. More contact with LTC staff and family members may be more difficult to arrange, but this may be a role for volunteers. The potential benefits and risks of adding new medications should be considered carefully. Research is needed on how best to implement these practices and on specific medications that may be associated with changes in severity of delirium symptoms in this population.

The authors have no financial or any other kind of personal conflicts with this paper. 
TABLE 1 Resident Baseline Characteristics (n=272)

\begin{tabular}{|ll|}
\hline Characteristic & n (\%) \\
\hline Age & \\
\hline $65-79$ & $63(23.2)$ \\
\hline $80-89$ & $135(49.6)$ \\
\hline$\geq 90$ & $74(27.2)$ \\
\hline Sex & \\
\hline Female & $156(57.4)$ \\
\hline Male & $116(42.6)$ \\
\hline Time since admission, years & \\
\hline$<1$ & $94(34.6)$ \\
\hline$\geq 1$ & $178(65.4)$ \\
\hline Dementia & \\
\hline No & $94(34.6)$ \\
\hline Yes & $178(65.4)$ \\
\hline Hierarchic Dementia Scale score & \\
\hline Severe (0-39) & $42(15.4)$ \\
\hline Moderate (40-99) & $26(9.6)$ \\
\hline Mild (100-159) & $108(39.7)$ \\
\hline Minimal/none $(\geq 160)$ & $96(35.3)$ \\
\hline Charlson Comorbidity Index & \\
\hline $0-1$ & $64(23.5)$ \\
\hline $2-3$ & $127(46.7)$ \\
\hline $4-15$ & $81(29.8)$ \\
\hline Mini-Mental State Examination score \\
\hline$<10$ & $71(26.1)$ \\
\hline $10-30$ & $201(73.9)$ \\
\hline
\end{tabular}


TABLE 2 Descriptive Statistics of Exposures and Outcomes Over Time in the Study Sample $(\mathbf{n}=\mathbf{2 7 2})$

Exposure and Outcome Variable

\section{Mean \pm Standard Median}

Deviation

(Interquartile

Range)

Binary variables, \%*

Absence of reading glasses (if visually impaired) $\quad 24 \pm 34 \quad 5(0-40)$

Absence of hearing aids (if hearing impaired) $\quad 12 \pm 27 \quad 0$ (0-6)

Absence of aids to orientation $32 \pm 39 \quad 9(0-66)$

Absence of personal possessions $\quad 4 \pm 16 \quad 0(0-0)$

Absence of family member $\quad 82 \pm 30 \quad 100(75-100)$

Absence of sensory stimulation $\quad 20 \pm 25 \quad 9(0-31)$

Bed rail $\quad 23 \pm 34 \quad 0(0-49)$

Other restraint $\quad 21 \pm 35 \quad 0(0-36)$

Absence of glass of water $\quad 67 \pm 33 \quad 77(42-95)$

No visible staff member $\quad 31 \pm 27 \quad 28$ (6-50)

Continuous variables ${ }^{b}$

Number of weekly assessments [range: 2-24] $\quad 17.2 \pm 6.2 \quad 20.0(15-22)$

Number of environmental risk factors [range: 0-10] $3.2 \pm 1.1 \quad 3.0(2.4-3.7)$

Mean daily number of medications ${ }^{\mathrm{c}}$ [range 0-15] $\quad 6.7 \pm 3.6 \quad 6.5(4.2-9.1)$

Delirium Index (DI) score [range: 0-21] $\quad 6.6 \pm 4.7 \quad 5.7(2.5-10.1)$

Weekly DI change $^{\mathrm{d}}$ [range -21 to +21$] \quad 0.01 \pm 0.33 \quad 0.00(-0.09-0.10)$

${ }^{\mathrm{a}}$ For each resident, the longitudinal data are summarized with the proportion over the 24 weeks.

${ }^{\mathrm{b}}$ For each resident, the longitudinal data are summarized with the average over the 24 weeks (see statistical methods for more details).

${ }^{\mathrm{c}}$ For each resident, the mean daily number of medication during a week is computed

${ }^{\mathrm{d}}$ For each resident, the mean weekly change at time $\mathrm{t}=$ Delirium Index (DI)(t)-DI(t-1). The proportion of residents having at least one weekly DI change with: an increase of 1 point is 87\%; an increase of 2 points or more is $85 \%$; no change is $96 \%$; a decrease of 1 point is $88 \%$; a decrease of 2 points or more is $85 \%$. 
TABLE 3 Mean Weekly Change in Delirium Index by Exposure ${ }^{a}$ to the Environmental Risk Factors (n=4,665 Resident Weekly Assessments)

\begin{tabular}{|c|c|c|c|c|c|c|c|c|c|}
\hline & \multicolumn{3}{|c|}{ Increased exposure } & \multicolumn{3}{|c|}{ Decreased exposure } & \multicolumn{3}{|c|}{ Continued exposure } \\
\hline & $\%$ & Beta1 & Beta2 & $\%$ & Beta1 & Beta2 & $\%$ & Beta1 & Beta2 \\
\hline \multicolumn{10}{|l|}{ Risk factors } \\
\hline $\begin{array}{l}\text { Absence of reading } \\
\text { glasses }^{\text {e }}\end{array}$ & 5.5 & $0.30^{c}$ & $0.30^{c}$ & 5.3 & $-0.34^{b}$ & $-0.34^{b}$ & 17.2 & $0.13^{c}$ & $0.16^{b}$ \\
\hline $\begin{array}{l}\text { Absence of hearing } \\
\text { aids }\end{array}$ & 2.8 & -0.06 & 0.03 & 2.9 & $-0.27^{\mathrm{d}}$ & $-0.28^{\mathrm{d}}$ & 7.7 & 0.12 & 0.12 \\
\hline $\begin{array}{l}\text { Absence of aids to } \\
\text { orientation }^{\mathrm{e}}\end{array}$ & 5.1 & $0.26^{c}$ & 0.19 & 5.4 & -0.05 & -0.07 & 25.2 & $0.26^{b}$ & $0.13^{c}$ \\
\hline $\begin{array}{l}\text { Absence of personal } \\
\text { possessions }\end{array}$ & 1.2 & 0.24 & 0.13 & 1.1 & -0.09 & -0.16 & 2.8 & -0.01 & $-0.26^{\mathrm{d}}$ \\
\hline $\begin{array}{l}\text { Absence of family } \\
\text { member }^{\mathrm{e}}\end{array}$ & 6.0 & 0.03 & -0.06 & 6.0 & $-0.34^{b}$ & $-0.37^{b}$ & 75.7 & $-0.17^{c}$ & $-0.17^{c}$ \\
\hline $\begin{array}{l}\text { Absence of sensory } \\
\text { stimulation }\end{array}$ & 10.2 & 0.00 & 0.07 & 10.1 & 0.07 & 0.13 & 8.4 & 0.06 & $0.14^{\mathrm{d}}$ \\
\hline Bed rails $^{\mathrm{e}}$ & 4.1 & 0.16 & 0.13 & 4.1 & 0.10 & 0.09 & 18.7 & 0.06 & $0.14^{\mathrm{C}}$ \\
\hline Other restraint $^{\mathrm{e}}$ & 4.9 & 0.19 & $0.23^{\mathrm{d}}$ & 4.6 & $0.23^{\mathrm{d}}$ & 0.20 & 15.5 & $0.14^{\mathrm{c}}$ & $0.21^{b}$ \\
\hline $\begin{array}{l}\text { Absence of glass of } \\
\text { water }^{\mathrm{e}}\end{array}$ & 11.4 & $0.22^{c}$ & $0.25^{b}$ & 11.1 & 0.01 & 0.09 & 54.2 & $0.14^{c}$ & $0.16^{b}$ \\
\hline $\begin{array}{l}\text { Absence of staff } \\
\text { member }\end{array}$ & 13.6 & 0.05 & -0.03 & 13.4 & -0.01 & -0.06 & 13.6 & -0.05 & $-0.11^{d}$ \\
\hline
\end{tabular}

${ }^{\mathrm{a}}$ Increased exposure indicates presence of the risk factor only at time t. Decreased exposure indicates presence of the risk factor only at time $t-1$. Continued exposure indicates presence of risk factor at time $\mathrm{t}-1$ and $\mathrm{t}$. The reference category is absence of risk factors at times $\mathrm{t}-1$ and $\mathrm{t}$. Beta represents the estimate from the generalized linear model: mean weekly Delirium Index (DI) change for one category. Compared to the reference category (no exposure). Beta1 was adjusted for time and previous DI score. Beta2 was adjusted for time, previous DI score, baseline variables (age, sex, duration of residence, dementia, Charlson Comorbidity Index, and severe cognitive impairment stratum (Mini-Mental State Examination score<10)) and all 10 risk factors presented above.

${ }^{\mathrm{b}} \mathrm{p}<0.01 ;{ }^{\mathrm{c}} \mathrm{p}<0.05 ;{ }^{\mathrm{d}} \mathrm{p}<0.10$

${ }^{\mathrm{e}}$ Selected risk factors for environmental risk factor score.

Bold font represents statistically significant results at .05 level. 
McCusker et al.

TABLE 4 Mean Weekly Change in the Delirium Index (DI) ${ }^{\mathrm{a}}$ by Changes in the Number of Environmental Risk Factors and in Daily Number of Medications, Overall and Stratified by Dementia (n=4,665 Resident Weekly Assessments)

\begin{tabular}{|c|c|c|c|c|c|c|}
\hline \multirow[t]{3}{*}{ Change } & \multirow[t]{3}{*}{$\%$} & \multicolumn{2}{|c|}{ Overall $N=4,665$} & \multicolumn{2}{|c|}{ Stratified Analysis } & \multirow{3}{*}{$\begin{array}{l}\text { P- } \\
\text { Value }^{\mathrm{e}}\end{array}$} \\
\hline & & & & $\begin{array}{l}\text { Dementia } \\
\mathrm{n}=3,064\end{array}$ & $\begin{array}{l}\text { No dementia } \\
\mathrm{n}=1,601\end{array}$ & \\
\hline & & Beta1 & Beta2 & Beta2 & Beta2 & \\
\hline $\begin{array}{l}\text { Environmental risk factor } \\
\text { score (range 0-6) }\end{array}$ & & & & & & .66 \\
\hline Increase of 1 & 18.7 & $0.13^{\mathrm{d}}$ & $0.14^{\mathrm{C}}$ & $0.17^{\mathrm{d}}$ & 0.08 & \\
\hline Increase of $\geq 2$ & 4.2 & $0.37^{b}$ & $0.41^{b}$ & $0.46^{b}$ & 0.28 & \\
\hline Decrease of 1 & 19.7 & 0.04 & 0.05 & 0.04 & 0.07 & \\
\hline Decrease of $\geq 2$ & 4.1 & $-0.32^{\mathrm{C}}$ & $-0.28^{\mathrm{C}}$ & $-0.31^{\mathrm{d}}$ & -0.19 & \\
\hline No change (reference) & 53.3 & 0.00 & 0.00 & 0.00 & 0.00 & \\
\hline $\begin{array}{l}\text { Mean daily number of } \\
\text { medications }\end{array}$ & & & & & & .43 \\
\hline Increase of 1 & 14.0 & $0.14^{\mathrm{d}}$ & 0.11 & 0.13 & 0.09 & \\
\hline Increase of $\geq \mathbf{2}$ & 4.2 & $0.49^{b}$ & $0.51^{b}$ & $0.77^{b}$ & 0.05 & \\
\hline Decrease of 1 & 14.9 & 0.05 & 0.02 & 0.06 & -0.09 & \\
\hline Decrease of $\geq 2$ & 3.3 & -0.11 & -0.11 & -0.16 & -0.06 & \\
\hline No change (reference) & 63.6 & 0.00 & 0.00 & 0.00 & 0.00 & \\
\hline
\end{tabular}

${ }^{a}$ Beta represents the estimate from the generalized linear model: mean weekly Delirium Index (DI) change for one category compared with the reference category (no change). Beta1 was adjusted for time and previous DI score. Beta2 was adjusted for time, previous DI score, baseline variables (age, sex, duration of residence, dementia, Charlson Comorbidity Index, and severe cognitive impairment stratum (Mini-Mental State Examination score <10) and both predictors presented in the table. ${ }^{\mathrm{b}} \mathrm{p}<0.01 ;{ }^{\mathrm{c}} \mathrm{p}<0.05 ;{ }^{\mathrm{d}} \mathrm{p}<0.10$

${ }^{\mathrm{e}}$ Interaction between the exposure variable and dementia.

Bold font represents statistically significant results at .05 level. 


\section{REFERENCES}

1. McCusker J, Cole MG, Voyer P et al. Use of nurse-observed symptoms of delirium in long-term care: Effects on prevalence and outcomes of delirium. Int Psychogeriatr 2011;23:602-608.

2. Kiely DK, Bergmann MA, Murphy KM et al. Delirium among newly admitted postacute facility patients: Prevalence, symptoms, and severity. J Gerontol A Biol Sci Med Sci 2003;58A:M441-M445.

3. Marcantonio ER, Simon SE, Bergmann MA et al. Delirium symptoms in postacute care: Prevalent, persistent, and associated with poor functional recovery. $\mathrm{J}$ Am Geriatr Soc 2003;51:4-9.

4. Sandberg O, Gustafson Y, Brannstrom B et al. Prevalence of dementia, delirium and psychiatric symptoms in various care settings for the elderly. Scand J Soc Med 1998;26:56-62.

5. Fries BE, Mehr DR, Schneider D et al. Mental dysfunction and resource use in nursing homes. Med Care 1993;31:898-920.

6. Voyer P, Richard S, Doucet L et al. Detecting delirium and subsyndromal delirium using different diagnostic criteria among demented long-term care residents. J Am Med Dir Assoc 2009;10:181-188.

7. McCusker J, Cole MG, Voyer P et al. Prevalence and incidence of delirium in long-term care. Int J Geriatr Psychiatry 2011;26:1152-1161.

8. Kiely DK, Jones RN, Bergmann MA et al. Association between psychomotor activity delirium subtypes and mortality among newly admitted postacute facility patients. J Gerontol A Biol Sci Med Sci 2007;62A:174-179.

9. Kiely DK, Marcantonio ER, Inouye SK et al. Persistent delirium predicts greater mortality. J Am Geriatr Soc 2009;57:55-61.

10. Inouye SK, Charpentier PA. Precipitating factors for delirium in hospitalized elderly persons. Predictive model and interrelationship with baseline vulnerability. JAMA 1996;275:852-857.

11. McCusker J, Cole MG, Abrahamowicz M et al. Environmental risk factors for delirium in hospitalized older people. J Am Geriatr Soc 2001;49:1327-1334.

12. Han L, McCusker J, Cole MG et al. Use of medications with anticholinergic effect predicts clinical severity of delirium symptoms in older medical inpatients. Arch Intern Med 2001;161:1099-1105.

13. Cole MG, McCusker J, Voyer P et al. Symptoms of delirium occurring before and after episodes of delirium in older long-term care residents. J Am Geriatr Soc 2012;60:2302-2307.

14. Cole M, McCusker J, Voyer P et al. Subsyndromal delirium in older long-term residents: Incidence and risk factors. J Am Geriatr Soc 2011;10:1829-1836. 
15. McCusker J, Cole M, Voyer P et al. Development of a delirium risk screening tool for long-term care facilities. Int J Geriatr Psychiatry 2012;27:999-1007.

16. Inouye SK, VanDyck $\mathrm{CH}$, Alessi CA et al. Clarifying confusion: The Confusion Assessment Method, a new method for detection of delirium. Ann Intern Med 1990;113:941-948.

17. McCusker J, Cole MG, Bellavance F et al. Reliability and validity of a new measure of severity of delirium. Int Psychogeriatr 1998;10:421-433.

18. McCusker J, Cole MG, Dendukuri N et al. The Delirium Index: A measure of the severity of delirium: New findings on reliability, validity and responsiveness. J Am Geriatr Soc 2004;52:1744-1749.

19. Folstein MF, Folstein SE, McHugh PR. "Mini-mental state". A practical method for grading the cognitive state of patients for the clinician. J Psychiatr Res 1975;12:189-198.

20. Tombaugh TN, McIntyre NJ. The Mini-Mental State Examination: A comprehensive review. J Am Geriatr Soc 1992;40:922-935.

21. Kafonek S, Ettinger WH, Roca R et al. Instruments for screening for depression and dementia in a long-term care facility. J Am Geriatr Soc 1989;37:29-34.

22. Cole MG, Dastoor DP. A new hierarchic approach to the measurement of dementia. Psychosomatics 1987;28:298-304.

23. Boller F, Verny M, Hugonot-Diener L et al. Clinical features and assessment of severe dementia. A review. Eur J Neurol 2002;9:125-136.

24. Rönnberg L, Ericsson K. Reliability and validity of the Hierarchic Dementia Scale. Int Psychogeriatr 1994;6:87-94.

25. Charlson ME, Pompei P, Ales KL et al. A new method of classifying prognostic comorbidity in longitudinal studies: Development and validation. J Chron Dis 1987;40:373-383.

26. Field TS, Gurwitz JH, Avorn J et al. Risk factors for adverse drug events among nursing home residents. Arch Intern Med 2001;161:1629-1634.

27. Buntinx F, Niclaes L, Suetens C et al. Evaluation of Charlson's comorbidity index in elderly living in nursing homes. J Clin Epidemiol 2002;55:1144-1147.

28. Bravo G, Dubois M-F, Hébert R et al. A prospective evaluation of the Charlson Comorbidity Index for use in long-term care patients. J Am Geriatr Soc 2002;50:740-745.

29. Tao J. Mixed Models Analyses Using the SAS System: Course Notes. Cary, NC: SAS Institute, Inc., 2002.

30. Kutner MH, Nachtsheim C, Neter J et al. Applied Linear Regression Models, 4th Ed. New York: McGraw Hill College, 2003.

31. Craney TA, Surles JG. Model-dependent variance inflation factor cutoff values. Qual Eng 2002;14:391-403. 
32. Voyer P, Richard S, Doucet L et al. Examination of the multifactorial model of delirium among long-term care residents with dementia. Geriatr Nurs 2010;31:105-114.

33. Voyer P, Richard S, Doucet L et al. Precipitating factors associated with delirium among long-term care residents with dementia. Appl Nurs Res 2011;24:171-178.

34. Hoffman NB. Dehydration in the elderly: Insidious and manageable. Geriatrics 1991;46:35-38.

35. Burkhalter CL, Allen RS, Skaar DC et al. Examining the effectiveness of traditional audiological assessments for nursing home residents with dementiarelated behaviors. J Am Acad Audiol 2009;20:529-538.

36. O’Mahony R, Murthy L, Akunne A et al. Synopsis of the National Institute for Health and Clinical Excellence guideline for prevention of delirium. Ann Intern Med 2011;154:746-751. 


\section{Appendix 1: Measurement and Analysis of Nonmodifiable Time-Dependent Variables (Vital Signs, Laboratory Tests, X-Rays, Medical and Nursing Problems, and Hospital Events)}

Time-Dependant Variable

\section{Abnormal Cutoff}

A) Vital signs

1) Temperature:

$>38^{\circ} \mathrm{C}$ (rectal)

2) Respiratory rate per minute

$<12$ or $>24$

3) Pulse oximetry, \%)

$<92$

4) Pulse rate per minute

$<55$ or $>94$

5) Systolic arterial pressure, $\mathrm{mmHg}$

$<90$ or $>139$

6) Diastolic arterial pressure, $\mathrm{mmHg}$

$<51$ or $>89$

7) Summary variable for vital signs

Any abnormal results detected from A1 to A6 (yes or no)

B) Laboratory test results

1) Hemoglobin: $\quad<100 \mathrm{~g} / \mathrm{L}$

2) Hematocrit, \%)
Male
$<0.37$ or $>0.47$

Female

$<0.42$ or $>0.52$

3) Platelets $/ \mathrm{L} \times 10^{9}$

$<100$ or $>400$

4) White blood count $/ \mu \mathrm{L} \times 10^{9}$ in $\quad<4.8$ or $>12$

1,000 s

5) Absolute neutrophil count, $\mathrm{mmol} / \mathrm{L} \quad<1.5$ or $>8.0$

6) Serum sodium A, mmol/L $\quad<130$ or $>150$

7) Serum potassium, $\mathrm{mmol} / \mathrm{L} \quad<3$ or $>6$

8) $\mathrm{BUN}, \mathrm{mmol} / \mathrm{L} \quad>8.5$

9) Serum creatinine, $\mu \mathrm{mol} / \mathrm{L} \quad<44$ or $>150$

10) $\mathrm{BUN} /$ creatinine ratio $>0.1$

11) Glucose, $\mathrm{mmol} / \mathrm{L} \quad<3$ or $>12$

12) Alanine aminotransferase, IU/L $\quad<5$ or $>60$

13) Serum albumin $\mathrm{g} / \mathrm{L} \quad<32$

14) Urinary culture positive

15) Summary variable for laboratory Any abnormal results detected from B1 to B14 (yes or tests no)

C) Chest X-rays

1) Chest X-rays Done or not done

D) Medical problems extracted from medical progress notes

1) Cardiovascular Myocardial infarction; coronary artery disease; congestive heart failure; peripheral vascular disease; valvular disease; arrhythmia, pacemaker, or defibrillator; hypertension or high blood pressure

2) Neurological Cerebral vascular accident, hemiplegia, chronic cognitive deficit, Parkinson's disease, other degenerative disease, other neurological not degenerative

3) Pulmonary Chronic pulmonary disease

4) Gastrointestinal

Liver disease, peptic ulcer disease, inflammatory bowel disease, constipation, fecaloma 
McCusker et al.

5) Renal and urinary

Moderate or severe renal disease, chronic renal failure, other renal disease, indwelling bladder catheter, urinary retention, other urinary problems

6) Endocrine Diabetes mellitus, thyroid disorder, other endocrine disorders

7) Neoplasms Solid tumor, leukemia, lymphoma

8) Visual or hearing impairment Severe or other impairment (e.g., cataracts not operated, glaucoma, myopia, presbyopia, deafness, presbycusis)

9) Pressure sore

10) Injuries or accidents Head trauma, fall with or without fracture, other fracture, injury

11) Infection

Fever, septicemia, pneumonia, respiratory infection, renal-urinary infection, gastrointestinal infection, skin infection, other infection

12) Surgery Any surgery

13) Any physical problem Any condition from D1 to D12 (yes or no)

E) Mental problems extended from medical progress notes

1) Depressive disorder or other Depressive disorder, mood disorder or mood episode, depression not specified

2) Anxiety Anxiety disorder or not specified

3) Other Substance abuse, other mental and/or behavioral problems

4) Any mental problem Any condition from E1 to E3 (yes or no)

F) Problems extracted from nursing notes.

1) Physical change

Any of the following (yes or no): Neurological ,sensory, cardiovascular, pulmonary, gastroenterological, urorenal, endocrine, skin, pain, injury, fall, fracture, constipation, aggression, uncooperative

2) Change in autonomy Any of the following (yes or no): Decreased mobility, poor balance, dressing, feeding, grooming, continence, assisted transfer, palliative care

G) Hospitalization or emergency department visit

1) Hospital visit Any of the following (yes or no): Discharge from acute hospitalization or emergency visit

BUN= blood urea nitrogen

\section{Definition of the change for the variables in section $A$ to $\mathbf{C}$}

Step 1: Each condition was coded at week " $t$ " using a hierarchical approach:

1) Abnormal: if any abnormal results were detected during the week

2) Normal: if all the results during the week were normal

3) Not done: no test results were available for the week.

Step 2:

a) The change between two specific weeks ( $t-1)$ and $(t)$ for the summary conditions A7 and B15 was defined as follows: 
1) (not done or normal) to abnormal

2) not done to normal

3) normal to not done

4) abnormal to (not done or normal)

5) no change [reference category]

b) The change between two specific weeks (t-1) and (t) for C1 was defined as follows:

1) not done (t-1) and done $(\mathrm{t})$

2) done $(\mathrm{t}-1)$ to not done $(\mathrm{t})$

3) not done at both times [reference category]

Step 3: Only the variables in step 2 were retained for the final model

\section{Definitions of the variables in sections D to G}

For D13, E17, F1, or F2, only the first date of the occurrence of a clinical condition was retained.

These variables occurring in the time interval $[t-1, t]$ were linked to the outcome (DI change: DI(t)-DI(t-1)) at time t.

For G1, each event was linked to the outcome (similar approach to that above).

Only D13, E17, F1, F2, and G1 were retained in the final model.

\section{Sensitivity analyses}

These analyses were repeated using less-strict criteria (below) to define abnormal results in sections $\mathrm{A}$ and $\mathrm{B}$, with similar results.

A6: $<60$ or $>89 \mathrm{mmHg}$

B1: $<140 \mathrm{~g} / \mathrm{L}$

B3: $<130$ or $>400 \times 10^{9} / \mathrm{L}$

B4: $<4.8$ or $>10.8 \times 10^{9} / \mu \mathrm{m}$

B6: $<135$ or $>145 \mathrm{mmol} / \mathrm{L}$

B7: $<3.5$ or $>5 \mathrm{mmol} / \mathrm{L}$

B11: $<3$ or $>6 \mathrm{mmol} / \mathrm{L}$ 